\title{
An integrated model for defining the scope of psychogeriatrics: the five Cs
}

Psychogeriatrics is a specialty defined by its many contrasts and complexities. Over-reliance on biological models sometimes artificially narrows the breadth and content of psychogeriatric research, educational programs, service delivery and management models to the detriment of patient care. This guest editorial proposes a conceptual model that defines the scope of the field and provides a structure that overlays standard approaches to diagnosis and formulation. Five key defining elements of psychogeriatrics - the five "Cs" - are explored: complexity, chronicity, comorbidity, continuity and context.

\section{Complexity}

Psychogeriatric practitioners recognize that multiple interacting factors create a complex picture in virtually every case. An illustration of the degree of complexity inherent in psychogeriatrics is the intriguing relationship among life experience, stress, personality and psychological developmental factors, and core physiological and neuropathological processes. An array of relatively recent data supports the importance of refining a "complex model" of formulation and intervention which may be especially relevant to the elderly.

Old age is inevitably associated with various stressful life events and it is well recognized that many elders become depressed under their impact. Despite the fact that depression is arguably the most common psychiatric disturbance of old age I suggest that it is surprisingly uncommon, when one considers the array of challenges posed by old age. Because the majority of elders do not become depressed, it stands to reason that the stressors associated with aging only induce depression if mediated by other vulnerability factors in a given individual. The data suggest that these factors include the complex interplay of three key elements that appear to mediate increased vulnerability to depressive symptoms: physiological and immunological reactions, personality structure, and early psychological development.

Stress has wide ranging physiological and immunological effects on the vulnerable body.

First published online 9 June 2009.
Mood disorders, closely associated with stress, also are associated with increased allostatic load including increased adrenocortical activity, raised concentrations of insulin growth factor (IGF)-1, initiation of the inflammatory response, impaired immunity, induction of arteriosclerosis, increased obesity, bone demineralization, and atrophy of brain cells (McEwen, 2004). Animal data suggest that stress-mediated alterations in brain-derived neurotrophic and corticotropin releasing factors may also be associated with major depressive disorder and anxiety (Anisman, 2009). A particularly interesting line of investigation that has the potential to integrate links among personality, physiology and psychopathology is the association of depression with elevated serum pro-inflammatory cytokine levels (especially interleukin-1 $\beta$, interleukin-6, tumour necrosis factor- $\alpha$ and interferon- $\alpha$ ) (Brebner et al., 2000; Anisman, 2009).

There is reason to be particularly concerned about the vulnerability of elders to cytokine activity and immunological factors. Cytokines are stimulated not only by stress but also by inflammatory disorders and diseases especially common in elders including cardiovascular disease, osteoporosis, arthritis, type 2 diabetes, various cancers, lymphoproliferative diseases, Alzheimer's disease and some treatments such as interferon in cancer (Anisman et al., 2008; Dantzer and Kelly, 2007; Konsman et al., 2002). Depression is associated with increased cardiovascular disease, although the underlying mechanisms are not well understood. Inflammatory markers such as interleukin 6 and C-reactive protein are associated with age and predict functional decline, mortality, decreased functional status, and disability (Hamerman et al., 1999; Currie et al., 1994; Cohen et al., 1997; Pieper et al., 2000; Ferrucci et al., 1999; Harris et al., 1999). Distress or depression seems to be associated with greater immunological impairments in older adults (Graham et al., 2006; Kiecolt-Glaser et al., 2003). IL-6 may be a marker for impending deterioration in health status in older adults and IL-6 levels increase with age (Ferrucci et al., 1999). Aging spousal caregivers of individuals with Alzheimer's disease are at greater risk of impaired immunological function particularly if they have poor levels of social support (KiecoltGlaser et al., 1991; Bauer et al., 2000). 
Beyond depression and stress per se, there is an association between elevated cytokine levels and personality traits that predispose to negative emotional states such as pessimism, sense of meaninglessness and negative self perceptions (Kiecolt-Glaser et al., 2002; Maruta et al., 2000). The mechanism of how a personality-emotionimmune interaction works is not entirely clear yet but some data support the hypothesis that personality structure may predispose an individual to greater relative positive or negative emotions including depression, which in turn influence immune function reflected in immune cell counts in peripheral blood (Segerstrom, 2000). For example, one personality factor that is associated with negative emotional states and that may be particularly important in predicting late-onset depression is sociotropy, defined as an unusual need for approval and reassurance in interpersonal relationships. This trait combined with negative interpersonal events, poorer physical functioning and medical illness has been found to be a primary predictive factor for late life depression (Mazure et al., 2002).

Data from other studies help further specify the nature of these positive and negative emotional states. Positive emotions that may be an indicator of resilience in the face of stress and adversity include the following: the ability to make a positive reappraisal of stressful life experiences and to find meaning in life and events, developing positive illusions about the self and situational optimism (Maruta et al., 2000). Some have hypothesized that these characteristics can have a positive effect on health outcomes possibly mediated through endocrine and immune mechanisms (KiecoltGlaser et al., 2002). Conversely, data support the contention that pessimism (vs. optimism) may predict physical illness and mortality in initially healthy adults (Maruta et al., 2000).

Taken together this research suggests a model of depression based on a highly complex feedback system in which negative emotional states associated with less adaptive personality structures induce physiological and neuroimmunological changes that in turn further exacerbate the negative emotional states. A complex cascade can now be hypothesized for late life depression as follows: elders are at risk of depressive symptoms when stress - i.e. their environment, illness or life events - interact with personality-based vulnerabilities to induce negative emotions causing demoralization and fear. In turn, these emotional states alter immune responses to produce proinflammatory changes that raise cytokine levels and induce depressive symptoms. Alternatively or concurrently, certain illnesses like diabetes or heart disease (Anisman, 2009) may themselves be both pro-inflammatory (raising cytokines) and inducing negative emotional states which then go on to auto-induce further depression.

This growing array of data illustrates how important it is to consider complexity when clinically evaluating depressive symptoms in elders. Training and practice must adapt current diagnostic systems to capture the full array of complex elements which the psychogeriatrician must address in each case, i.e. personality and development, physical health, psycho-neuroimmunological function, and sources and psychological impact of stress.

\section{Chronicity}

Old age is characterized both by longstanding problems and by diseases which, once they start in old age, often become chronic. The line between acute reversible illness and chronic irreversible illness is often blurred in elders, as illustrated by this clinical vignette.

A 78-year-old man was referred for management of unresolved grief following his wife's death. Examination revealed mild impairments in memory and some personality changes. From being a dominant rather aggressive business man he had become uncertain, worried and vulnerable. He spoke of mild, but clearly perceptible, subjective problems in recent memory, concentrating on complex business matters that he had handled easily in the past, and very occasional lapses of orientation when driving for which he could compensate with effort. Formal mental status testing showed mild memory and some mild executive dysfunction which did not cross the threshold for dementia. However, these symptoms pointed to early cognitive decline and a diagnosis of mild cognitive impairment of uncertain origin. If the diagnosis was accurate the likelihood was that he would progress to some form of dementia. In other words, this patient, while referred for an acute depressive response to a life event, had concurrently entered the domain of chronic and incurable illness.

Dementia is an obvious example of chronicity, but there are other less obvious pathways to chronic illness in elders, for example anxiety. Schuurmans et al. (2005) reported that, after six years of follow-up, about $70 \%$ of geriatric patients continued to meet criteria for full-blown or subsyndromal anxiety disorder. Chronic anxiety in elders has important implications, including increased disability and mortality rates, impaired quality of life and psychosocial functioning (de Beurs et al., 1999; Beekman et al., 1999; Wetherell 
et al., 2004; van Hout et al., 2004). Depression in late life often has a prolonged course and variable treatment outcome in late life. Clinicians are given conflicting guidelines on how long to continue maintenance therapy which for some elders can be very prolonged. For example, Reynolds et al. (2006) found that at least a two-year maintenance period is important to prevent relapse.

While chronicity is obviously an important consideration in many patients our clinical and research paradigms generally do not systematically address its key components such as suffering or demoralization. Chronic illness implies chronic suffering that arises especially from conditions that induce demoralization and hopelessness, anxiety, withdrawal and loneliness. These emotional reactions often do not reach thresholds for DSM diagnosis but represent significant burden. The rather abstract and philosophical nature of suffering makes it difficult to operationalize, but it is important to be able to measure suffering if we are to address it. Attempts have been made to do so. Gurland et al. (1997), for example, developed the Index of Affective Suffering, a scale that measures subjective symptom severity which is highly relevant to chronic illness. The elements of the index include "intensity" and "extensity" of distress (including number and variety of life events, duration and frequency of episodes, and activities that are pervaded by distress). The scale defines levels of severity: Minor/moody, Background, Dominant, Desperate, Intolerable. It is notable that the discourse and focus of this scale differ from the phenomenologically based discourse of the standard diagnostic manuals.

Chronicity raises many therapeutic questions such as how long should the patient be followed, what is the best array of treatments to produce continued improvement in a given condition, which symptoms can be treated and which are unlikely to change? By adding the perspective of chronicity to clinical formulations important practical implications emerge. For example, chronicity redefines criteria and measures of outcomes of intervention, such as degree of amelioration of suffering in addition to symptom relief.

To incorporate chronicity systematically into routine clinical practice and formulation means adding some concepts and practices to training programs and recognizing that many training programs are not well designed to teach about chronicity. For example, supervisors will readily recognize that training rotations often are of short duration and follow-up of patients after initial evaluation is often inconsistent or non-existent.
Because of relatively short rotations on a given service, medical residents of various specialties trained in North American programs often get experience in initiating treatment but disturbingly little experience in maintaining and adjusting it. Psychogeriatric training requires a stronger emphasis on monitoring, adjusting and integrating interventions over time.

Psychogeriatric clinical training also needs to address the professional's reactions to confronting chronicity. If, in the face of chronic illness, one loses hope or is intolerant of dealing with problems that cannot be cured but only endured, then there is a danger of favoring dealing with problems crosssectionally or focusing only on symptoms that can be cured rather than those to which one must adapt. In doing this clinicians are in danger of abandoning many patients to their suffering.

Redefinition of psychogeriatric models that takes chronicity into account requires a more refined evidence-base for the management of chronicity to improve treatment and service delivery models and research design. Table 1 outlines the key elements of such a model.

\section{Comorbidity}

Psychogeriatric clinical care is characterized by multiple interacting concurrent illnesses and treatment modalities. This truth becomes evident from the epidemiology of psychogeriatric disorders. The greatest prevalence of mental disorders in the elderly is found in medical clinics and hospitals. Comorbidity is illustrated by looking at rates of depression in various medical settings and disease associations as illustrated in Table 2 .

Not all comorbidity is alike or has a significant impact on elders. For example, Oslin et al. (2002) suggested that chronic illnesses which produce functional decline or disability are the most likely form of comorbidty to negatively affect the treatment and outcome of depression. Arthritis is particularly implicated. Conversely, depression can lead to poorer outcomes for specific chronic medical disorders such as diabetes mellitus and myocardial infarction, although the mechanisms are not clear, perhaps related to poor self care, nutrition and adherence (Oslin et al., 2002). A similar conclusion can be derived from the work of Rovner et al. (2007) whose results suggest that the psychological impact of macular degeneration varies according to the meaning and import of the visual decline on the aging person.

The nature of depression associated with disability is perhaps best understood as demoralization 
Table 1. The key elements in the management of chronicity to improve treatment and service delivery

1. Define the point at which "chronic" models of care should be initiated

2. Understand and research the process of change within chronic disease entities over time. e.g. mortality health and psychiatric morbidity in grief and bereavement (Stroebe et al., 2007)

3. Delineate predictors of chronicity e.g. executive dysfunction subtype of depression (Kalayam and Alexopoulos, 1999; Alexopoulos et al., 2004)

4. Systematically define the stages of clinical change in chronic states

5. Define: when and how to intervene; when not to intervene in the natural course of the disease; when and how to move from active care to maintenance to discontinuation;

6. Define, measure and manage suffering

7. Determine need for and timing of long-term protective measures

8. Manage caregiver burden (Gitlin et al., 2003; Mittelman et al., 2008)

9. Address legal issues associated with chronicity, e.g. competency, power of attorney, living will, decision making, testamentary capacity, driving.

10. Determine cost- and clinically-effective frequency and duration of follow-up.

11. Define prognosis measures on the expectable course over time.

Table 2. Rates of comorbid depression in various settings and diseases

- $\quad$ Long term care settings $-50 \%$

- Primary care clinic settings - 6-25\%

- Hospitals - 5-13\% MD

- Outpatient medical settings - 3-16\% minor depression; up to $64 \%$ depressed mood

- Alzheimer's disease - minor depression and depressive symptoms reported in $20-50 \%$ of cases

- Parkinson's disease $-25-70 \%$

- Diabetes - 15-20\%

- Post myocardial infarction - 20-30\% major depression; 25\% minor depression

- $\quad$ CVA - $25 \%$

- cancer $25-50 \%$

$\mathrm{MD}=$ major depression; $\mathrm{CVA}=$ cerebrovascular accident.

Sources: Table from Sadavoy, 2004. See also Cummings and Masterman, 1999; Lyketsos et al., 2000; Devanand, 1997; Harwood et al., 1999.

(as opposed to depressive disorder) and, as noted earlier, may be most appropriately characterized by the level of affective suffering rather than the diagnostic category or symptom profile. This position is supported by the work of Newmann et al. (1996) who examined the results obtained by using different measures of depression. They suggested that depressive symptom scales produce two groups of depressive symptoms: a "depressive syndrome" which is less common in the elderly and a "depletion syndrome" which is more common. The depletion syndrome is characterized by loneliness, lack of energy and sleep disturbance. Flint (2002) incorporates this type of thinking when he suggests that a dimensional rather than a categorical threshold is a better model for describing depressive symptoms in elders and that a dimensional approach may provide a "framework for studying the complex interactions between depression, anxiety, medical illness, cognitive impairment, personality factors and life stress." If this is true then treatment, at a minimum, likely requires both somatic therapy and psychotherapy to restore self esteem, find adaptive solutions, reestablish sense of mastery or control and help preserve engagement. Incorporating comorbidity into the formulation requires tailoring of educational programs to help students learn how to assess comorbidity and intervene in a specific fashion, including appropriate integration of consultative and support disciplines. The elements of comorbidity define the reciprocal importance of the comorbid condition in the production and treatment of psychogeriatric disorders and the impact of psychogeriatric disorders on treatment of comorbid conditions. Factors relevant to comorbidity in psychogeriatric clinical, educational and research practice are summarized in Table 3.

\section{Continuity}

Continuity, as it is used here, refers to the concept that each elderly person is the product of the lifelong cumulative effect of their environmental, psychological and physiological development. Every aged person brings into old age the legacy of their lifestyle, capacity for forming and maintaining 
Table 3. Factors relevant to comorbidity

- Disease states that induce psychogeriatric symptoms, e.g. arthritis, diabetes, heart disease

- Disease states that affect or confuse diagnosis

- Disease states that affect treatment, e.g. Lewy body psychosis

- Psychogeriatric conditions that complicate medical/surgical management of disease state, e.g. delirium after surgery in demented patients; falling phobia after hip fracture

- Disease states that induce secondary demoralization, e.g. loss of highly valued capacities or induction of longstanding fears such as abandonment

- Assessment of the meaning and impact of comorbidities

- Intervention strategies for comorbid conditions

- Techniques to integrate consultative and support disciplines into the care team

Table 4. Elements to be assessed in the evaluation of continuity factors

- Ability to control and modulate emotions when under stress

- Capacity to feel supported by others

- Sense of control over destiny and future

- Self esteem and sense of value and importance to others

- Capacity to trust

- Abandonment fears and capacity to tolerate being alone

- Psychological tolerance of physical illness, e.g. helplessness and demoralization versus philosophical acceptance

- Reactions to death and loss

- Capacity for friendship

- Pattern of interest and involvement in versus withdrawal from life

relationships, genetic vulnerabilities, physical makeup and illnesses. Of particular importance are factors of psychological development and continuity. For example, personality evolves from the earliest childhood experiences and remains remarkably stable into old age, although certain traits may evolve as the individual adapts to contextual and interpersonal forces associated with aging (Roberts et al., 2006). The unconscious is commonly referred to as timeless and endures, albeit somewhat modified, into old age. Psychogeriatricians, to be most effective, must be alert to the influence of these lifelong factors on psychogeriatric illnesses and the capacity of the patient to adapt to late life illnesses without undue or overwhelming anxiety or depression. Moreover, personality traits such as conscientiousness, activity and emotional stability appear to have a direct impact not only on the capacity to adapt to aging but to longevity itself (Terracciano et al., 2008).

For example, a 68-year-old woman was referred for depression which developed after somewhat mutilating cancer surgery. On first impression, her symptoms were understandable as a reaction to a significant life stress. However, her intent in seeking out treatment was more subtle. First, she had a difficult time during the lead-up to and following her surgery especially associated with rather callous handling by one of her physicians. This experience in turn was associated with the quite sudden reemergence of long dormant memories of severe physical and emotional abuse at the hands of her parents leading to some depressive symptoms but, more importantly, to the return of severe post-traumatic stress disorder symptoms that had been well controlled through her adult life. The psychodynamics are complex, but the vignette succinctly illustrates the crucial importance of a longitudinal perspective on careful evaluation of the patient with attention to understanding the continuity of each patient's life and incorporating it into the therapeutic frame.

Personality-based factors have demonstrable impact on treatment outcome. Data suggest that personality disorders (PD) negatively impact the effectiveness of treatment (Karp et al., 1993; Thompson et al., 1988) and personality disorders contribute to functional disability after antidepressant treatment (Abrams et al., 1998). Cluster C personality disorders, i.e. avoidant, dependent, obsessive-compulsive, and self-defeating types, have been associated with less stable remission and with a slower response time to therapy (15 versus 10 weeks) (Morse et al., 2005).

To operationalize the concept of psychological continuity in the elderly requires training in how to acquire the data and techniques for using it. Table 4 presents an outline of elements to be assessed in the evaluation of continuity factors, while Table 5 presents elements that can help to operationalize continuity into psychogeriatric practice. 
Table 5. Elements that can help to operationalize continuity into psychogeriatric practice

- Psychological and personality-based factors: problem solving, optimism, conflicted relationships, early loss, trauma

- Lifestyle: smoking, medication, substance use, exercise, diet

- Sources of satisfaction: hobbies, work, activities

- Spirituality

- Intimacy, friendship, kinship patterns

- Adaptation to prior illness, disability: psychiatric or physical

Table 6. Contextual issues

\begin{tabular}{|c|c|}
\hline ISSUES & EXAMPLES \\
\hline Social/support & $\begin{array}{l}\text { Perceived support is associated with the extent of caregiver burden and the } \\
\text { outcome of depression treatment (Cui et al., 2008); erosion of friendship } \\
\text { networks by death and disability (Sadavoy and Sadavoy, 2006) }\end{array}$ \\
\hline Occupational and economic & Forced retirement \\
\hline Physiological & $\begin{array}{l}\text { Drug dosing decisions; dementia, agitation and cerebrovascular risk factors } \\
\text { affecting decisions to use atypical antipsychotics }\end{array}$ \\
\hline Illness and concurrent disorders & $\begin{array}{l}\text { Hidden alcohol abuse confusing management and diagnosis of } \\
\text { psychogeriatric disorders }\end{array}$ \\
\hline
\end{tabular}

\section{Context}

Psychiatric illness and psychological disturbances in old age cannot be separated from the physical, psychological, social and environmental context within which that illness emerges. For example, while grief is an almost universal experience, the context of the grief can modify its expression. Aberrant grief is more likely to emerge if the loss occurs in the context of a prior psychologically conflicted relationship with the lost person associated with insecure attachment patterns in the bereaved (Shear and Shair, 2005). Such reactions may not be responsive to medication and often do not resolve simply with the passage of time, as does normal grief.

Environmental, social and physical contexts interact and different settings such as community, long-term care or an acute care hospital each imply different management issues and outcome profiles. An example would be the patient who developed a paranoid state in a long-term care (LTC) home and began to refuse clinical care leading to loss of diabetes control. In the general hospital the very rapid evaluation and management of physically ill elders may place the psychogeriatric patient at particular risk of being misdiagnosed and inappropriately treated. For example, an elderly man developed a clear delirium associated with pneumonia. The physician, who knew very little about the patient's prior state and life, insisted that there was no need to investigate the acute change in the patient's mental state stating that it was dementia that was being uncovered by the move to the hospital. Instead of investigating the delirium, this precipitous and wrong diagnosis led him to ask that an application to a LTC facility be initiated. Occupational and other key functions, such as driving, impact the context of illness in the elders: for example, the dementing surgeon who had to be compelled to stop practicing while helping him and his family to face giving up the career satisfaction, money, prestige and security that went with his high profile career. Table 6 lists key contextual issues to be considered in psychogeriatric formulation.

\section{Conclusion}

The practice of psychogeriatrics includes, but extends far beyond, the narrow confines of neuropsychiatry. Clinical models of effective diagnosis and management must be comprehensive and of broad enough scope to encompass all the factors that affect assessment, treatment and outcome. While the models used for general adults are often useful for elders they are also incomplete in defining the complexities that are especially relevant to clinical interventions for the elderly. In this guest editorial I have tried to expand these models by suggesting a more comprehensive approach which I believe is practical and can be operationalized and incorporated into training and clinical practice. This model suggests a matrix approach. Along one axis are the standard phenomenological diagnostic elements while along the other are the factors that 
elaborate special essential elements associated with the elderly - the five Cs of psychogeriatrics.

\section{Joel SADAvoy}

Professor and Sam and Judy Pencer and Family Chair in Applied General Psychiatry at the University of Toronto, and Head of Geriatric and Community Psychiatry Programs, Mount Sinai Hospital, Toronto, Canada Email: jsadavoy@mtsinai.on.ca

\section{References}

Abrams, R. C. et al. (1998). Personality disorder symptoms and functioning in elderly depressed patients. American fournal of Geriatric Psychiatry, 6, 24-30.

Alexopoulos, G. S., Kiosses, D. N., Murphy, C. and Heo, M. (2004). Executive dysfunction, heart disease burden, and remission of geriatric depression.

Neuropsychopharmacology, 29, 2278-2284.

Anisman, H. (2009). Cascading effects of stressors and inflammatory immune system activation: implications for major depressive disorder. Fournal of Psychiatry and Neuroscience, 34, 4-20.

Anisman, H., Merali, Z. and Hayley, S. (2008). Neurotransmitter, peptide and cytokine processes in relation to depressive disorder: comorbidity between depression and neurodegenerative disorders. Progress in Neurobiology, 85, 1-74.

Bauer, M. E., Vedhara, K., Perks, P., Wilcock, G. K., Lightman, S. L. and Shanks, N. (2000). Chronic stress in caregivers of dementia patients is associated with reduced lymphocyte sensitivity to glucocorticoids. Fournal of Neuroimmunology, 103, 84-92.

Beekman, A. T. F., Copeland, J. R. M. and Prince, M. J. (1999). Review of community prevalence of depression in later life. British fournal of Psychiatry, 174, 307311.

Brebner, K., Hayley, S., Zacharko, R., Merali, Z. and Anisman, H. (2000). Synergistic effects of interleukin-1, interleukin-6, and tumor necrosis factor: central monoamine, corticosterone, and behavioral variations. Neuropsychopharmacology, 22, 566-580.

Cohen, H. J. et al. (1997). The association of plasma interleukin-6 levels with functional disability in community-dwelling elderly. Fournal of Gerontology: Medical Science, 52A, M201-M208.

Cui, X., Lyness, J., Tang, W., Tu, X. and Conwell, Y. (2008). Outcomes and predictors of late-life depression trajectories in older primary care patients. American fournal of Geriatric Psychiatry, 16, 406-415.

Cummings, J. L. and Masterman, D. L. (1999). Depression in patients with Parkinson's disease. International fournal of Geriatric Psychiatry, 14, 711718.

Currie, M. S., Rao, K. M. K., Blazer, D. G., and Cohen, H. J. (1994). Age and functional correlations of markers of coagulation and inflammation in the elderly - functional implications of elevated cross linked fibrin degradation products (D-dimers). Fournal of the American Geriatrics Society, 42, 738-742.
Dantzer, R. and Kelley, K. W. (2007). Twenty years of research on cytokine-induced sickness behavior. Brain, Behavior and Immunity, 21, 153-160.

de Beurs, E. et al. (1999). Consequences of anxiety in older persons: its effect on disability, well-being and use of health services. Psychological Medicine, 29, 583-593.

Devanand, D. P. et al. (1997). The course of psychopathologic features in mild to moderate Alzheimer disease. Archives of General Psychiatry, 54, 257-263.

Ferrucci, L. et al. (1999). Serum IL-6 level and the development of disability in older persons. Fournal of the American Geriatrics Society, 47, 639-646.

Flint, A. (2002). The complexity and challenge of non-major depression in late life. American fournal of Geriatric Psychiatry, 10, 229-232.

Gitlin, L. N. et al. (2003). Effect of multicomponent interventions on caregiver burden and depression: the REACH multisite initiative at 6-month follow-up. Psychology and Aging, 18, 361-374.

Graham, J. E., Christian, L. M. and Kiecolt-Glaser, J. K. (2006). Stress, age, and immune function: toward a lifespan approach. Fournal of Behavioral Medicine, 29, 389-400.

Gurland, B. J., Katz, S. and Chen, J. (1997). Index of affective suffering: linking a classification of depressed mood to impairment in quality of life. American fournal of Geriatric Psychiatry, 5, 192-210.

Hamerman, D. et al. (1999). Emerging evidence for inflammation in conditions frequently affecting older adults: report of a symposium. Fournal of the American Geriatrics Society, 47, 1016-1025.

Harris, T. B. et al. (1999). Associations of elevated interleukin-6 and C-reactive protein levels with mortality in the elderly. American fournal of Medicine, 106, 506-512.

Harwood, D. G., Barker, W. W., Ownby, R. L. and Duara, R. (1999). Association between premorbid history of depression and current depression in Alzheimer's disease. Fournal of Geriatric Psychiatry and Neurology, 12, 72-75.

Kalayam, B. and Alexopoulos, G. (1999). Prefrontal dysfunction and treatment response in geriatric depression. Archives of General Psychiatry, 56, 713-18.

Karp, J. F. et al. (1993). Time-to-remission in late-life depression: analysis of effects of demographic, treatment, and life-events measures. Depression, 1, 250-256.

Kiecolt-Glaser, J. K., Dura, J. R., Speicher, C. E., Trask, O. J. and Glaser, R. (1991). Spousal caregivers of dementia victims: longitudinal changes in immunity and health. Psychosomatic Medicine, 53, 345-362.

Kiecolt-Glaser, J. et al. (2002). Emotions, morbidity, and mortality: new perspectives from psychoneuroimmunology. Annual Review of Psychology, 53, 83-107.

Kiecolt-Glaser, J. K., Preacher, K. J., MacCallum, R. C., Atkinson, C., Malarkey, W. B. and Glaser, R. (2003). Chronic stress and age-related increases in the proinflammatory cytokine IL-6. Proceedings of the National Academy of Science, 100, 9090-9095.

Konsman, J. P., Parnet, P. and Dantzer, R. (2002). Cytokine-induced sickness behaviour: mechanisms and implications. Trends in Neurosciences, 25, 154-159.

Lyketsos, C. G. et al. (2000). Mental and behavioral disturbances in dementia: findings from the Cache County Study on memory in aging. American fournal of Psychiatry, $157,708-714$. 
Maruta, T. et al. (2000). Optimists vs. pessimists: survival rate among medical patients over a 30 -year period. Mayo Clinic Proceedings, 75, 140-143.

Mazure, C., Maciejewski, P. K., Jacobs, S. C. and Bruce, M. L. (2002). Stressful life events interacting with cognitive/personality styles to predict late-onset major depression. American fournal of Geriatric Psychiatry, 10, 297-304.

McEwen, B. S. (2004). Protection and damage from acute and chronic stress: allostasis and allostatic overload and relevance to the pathophysiology of psychiatric disorders. Annals of the New York Academy of Science, 1032, 1-7.

Mittelman, M., Brodaty, H., Wallen, A. and Burns, A. (2008). A three-country randomized controlled trial of a psychosocial intervention for caregivers combined with pharmacological treatment for patients with Alzheimer disease: effects on caregiver depression. American fournal of Geriatric Psychiatry, 16, 893-904.

Morse, J., Pilkonis, P., Houck, P., Frank, E. and Reynolds, C. (2005). Impact of Cluster C personality disorders on outcomes of acute and maintenance treatment in late-life depression. American fournal of Geriatric Psychiatry, 13, 808-814.

Newmann, J. P., Klein, M., Jensen, J. E. and Essex, M. J. (1996). Depressive symptom experiences among older women: a comparison of alternative measurement approaches. Psychology and Aging, 11, 112-126.

Oslin, D., Datto, C., Kallan, M., Katz, I., Edell, W. and ten Have, T. (2002). Association between medical comorbidity and treatment outcomes in late-life depression. Fournal of the American Geriatrics Society, 50, 823-828.

Pieper, C. F. et al. (2000). Age, functional status and racial differences in plasma D-dimer levels in community-dwelling elders. Fournal of Gerontology: Medical Science, 55A, M649-M657.

Reynolds, C. et al. (2006). Maintenance treatment of major depression in old age. New England Fournal of Medicine, 354, 1130-1138.

Roberts, B., Walton, K. and Viechtbauer, W. (2006). Patterns of mean-level change in personality traits across the life course: a meta-analysis of longitudinal studies. Psychological Bulletin, 132, 1-25.

Rovner, B. W., Casten, R. J., Hegel, M. T., Hauck, W. W. and Tasman, W. S. (2007). Dissatisfaction with performance of valued activities predicts depression in age-related macular degeneration. International fournal of Geriatric Psychiatry, 22, 789-931.

Sadavoy, J. (2004). Psychotropic Drugs and the Elderly: Fast Facts. New York: WW Norton.

Sadavoy, J. and Sadavoy, S. (2006) Friendship in old age. In R. Cohen, G. Pollack and R. Schulman (eds.), Friends and Friendship. Madison, CT: Psychosocial Press.

Schuurmans, J. et al. (2005). The outcome of anxiety disorders in older people at 6-year follow-up: results from the Longitudinal Aging Study Amsterdam. Acta Psychiatrica Scandinavica, 111, 420-428.

Segerstrom, S. C. (2000). Personality and the immune system: models, methods, and mechanisms. Annals of Behavioral Medicine, 22, 180-190.

Shear, K. and Shair, H. (2005). Attachment, loss, and complicated grief. Developments in Psychobiology, 47, 253-267.

Stroebe, M., Schut, H. and Stroebe, W. (2007). Health outcomes of bereavement. Lancet, 370, 9603.

Terracciano, A., Löckenhoff, C., Zonderman, A., Ferrucci, L. and Costa, P. (2008) Personality predictors of longevity: activity, emotional stability, and conscientiousness. Psychosomatic Medicine, 70, 621627.

Thompson, L. W., Gallagher, D. and Czirr, R. (1988). Personality disorder and outcome in the treatment of late-life depression. Fournal of Geriatric Psychiatry, 21, 133-153.

van Hout, H. P. et al. (2004). Anxiety and the risk of death in older men and women. British fournal of Psychiatry, 185, 399-404.

Wetherell, J. L., Thorp, S. R., Patterson, T. L., Golshan, S., Jeste, D. V. and Gatz, M. (2004). Quality of life in geriatric generalized anxiety disorder: a preliminary investigation. Fournal of Psychiatric Research, 38, 305-312. 